\title{
16 Years of RoboCup Rescue
}

\author{
Raymond Sheh $^{1} \cdot$ Sören Schwertfeger $^{2} \cdot$ Arnoud Visser $^{3}$ (1)
}

Received: 6 May 2016/Accepted: 15 July 2016/Published online: 16 August 2016

(c) The Author(s) 2016. This article is published with open access at Springerlink.com

\begin{abstract}
The RoboCup Rescue competitions have been initiated in 2000. To celebrate 16 years of research and development in this socially relevant initiative this article gives an overview of the experience gained during these competitions. This article provides an overview the stateof-the-art and the lessons learned from the RoboCup Rescue competitions.
\end{abstract}

\section{Introduction}

The urban search and rescue (USAR) scenario offers a great potential to inspire and drive research in multi-agent and multi-robot systems. In this article we like to introduce the RoboCup Rescue leagues, which are respectively the Rescue Robot League (RRL) and the Rescue Simulation League (RSL) [1, 2].

Disaster mitigation is an important social issue involving large numbers of heterogeneous agents acting in hostile environments. The associated Urban Search and Rescue (USAR) scenarios have great potential for inspiring and driving research in both multi-agent and multi-robot systems. Since the circumstances during real USAR missions are extraordinarily challenging [3], benchmarks based on them, such as the RoboCup Rescue competitions, are ideal for assessing the capabilities of intelligent robots. Robots that can navigate through affected areas after a disaster,

Arnoud Visser

A.Visser@uva.nl

Curtin University, Bentley, Australia

2 ShanghaiTech University, Shanghai, China

3 Universiteit van Amsterdam, Amsterdam, The Netherlands most likely will also be capable of negotiating the very same environment under normal circumstances. If robots are able to recognize humans entombed under piles of rubble of collapsed buildings, they will also be able to recognize them within their natural environment. The goal of the RoboCup Rescue competitions is to compare the performance of different algorithms for coordinating and controlling teams of either robots or agents performing disaster mitigation. By their nature, the USAR scenarios demand solutions for the coordination of large and distributed teams of heterogeneous robots.

In the remainder of this paper first the developments in the Rescue Robot League (Sect. 2) are highlighted, followed by the development in the Rescue Simulation League (Sect. 3).

\section{Rescue Robot}

The RoboCup Rescue Robot League (RRL) is a community of teams that make use of competitions, rescue camps and summer schools to advance the state of response robotics. Through the rescue competition the league is encouraging teams to work on robotic systems for USAR scenarios and providing opportunities to compare their solutions with other teams and get feedback from other experts and end-users. Figure 1 shows the community of scientists, engineers and robots present at the 2016 RoboCup RescueWord championship, organized in Leipzig, Germany.

\subsection{Competition Structure}

The RoboCup Rescue Robot League is a points scoring competition. In general, this consists of a search task within 


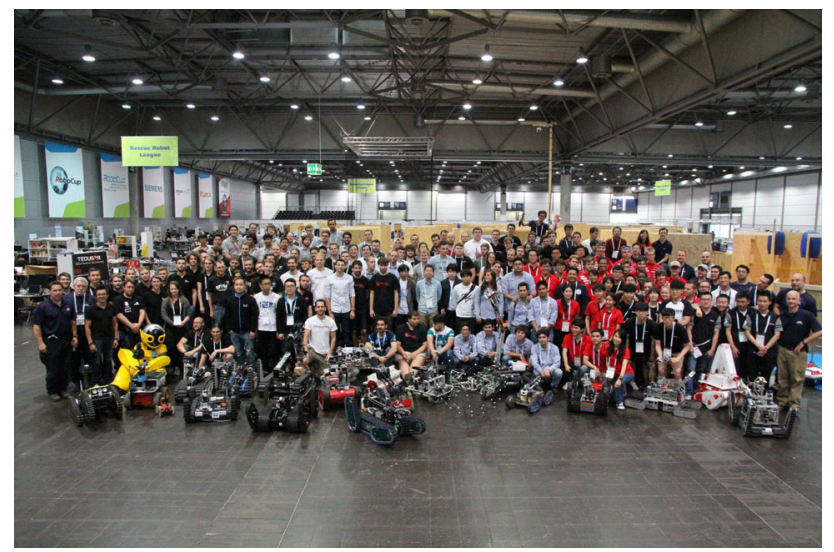

Fig. 1 Robots, teams and organizers at the RoboCup Rescue 2016 World Championships in Leipzig, Germany. These teams represent the best from the regional opens around the world.

an arena consisting of the standard test method apparatuses. The teams aim to reach and survey simulated victims within this arena and, in the process, overcome the various test methods. Over time, additional tasks such as autonomy, mapping and manipulation tasks have been added to the scoring metric to reflect improving capabilities and new focus areas.

Such a structure has several benefits. It helps to foster the spirit of collaboration, where all teams work on the common goal of the league, by specifically avoiding placing teams in an adversarial position. It contributes to fairness, since teams have the same challenges at their disposal. The use of standard test methods allows teams to analyze their successes and failures in a scientifically rigorous and reproducible manner, helping them to further improve their capabilities. It also allows for direct comparisons between the performance of robots within the competition and those in other settings.

One particular feature of the RoboCup Rescue Robot League is that it encourages the participation of not only the teams that are able to do well overall across the various tasks, but also teams that specialize in particular challenges of the application. These include advanced mobility, autonomous operations, mapping, manipulation and confined space operations. The use of distinct test methods to build the arena, combined with the ability of teams to choose their path, allows teams to focus their missions on their areas of strength. For example, teams with advanced mobility capabilities can spend their efforts on points in the arena that are dominated by mobility challenges. In contrast, teams that focus on manipulation can stick to areas of the arena with less challenging terrain but where collecting points requires dexterous robot arms to observe and manipulate objects.

Beyond the ability to demonstrate these specialized capabilities, the League recognizes that teams with superior capabilities in these niche areas do not necessarily have the ability to produce an entry that will perform well in the overall competition. To encourage these teams to compete and share their developments, the League tracks not only overall performance but also points earned in these specific capabilities. These points are combined with additional tests to evaluate these capabilities in isolation to determine the winners of the Best-in-Class awards. Past winners for the Best-in-Class awards appear in the RoboCup Rescue wiki. ${ }^{1}$

Other features of the competition structure include the splitting of the competition into preliminary and final rounds. All teams are guaranteed a set number of opportunities to run in the preliminaries, allowing all teams to demonstrate their capabilities in front of an audience of their peers. The number of teams that progress to the finals depends on the score distribution. The worst qualified team should be clearly better than the best team that failed to qualify. Teams that specialize tend to fail to qualify as their more specialized focus places them at a disadvantage. Therefore, the League encourages teams that did qualify, which tend to be more general in nature, to incorporate a team that did not qualify but who demonstrated superior performance in an area that they lack. The combined team progresses as one and any awards are given to both teams. This mechanism promotes collaboration between teams, helping to disseminate Best-in-Class capabilities throughout the League. Salient examples include mapping algorithms such as HectorSLAM [4].

The standard test methods, as defined by the $\mathrm{DHS}^{2}$ $\mathrm{NIST}^{3}-\mathrm{ASTM}^{4}$ International Standard Test Methods for Response Robots ${ }^{5}$ [5], are used inside the RRL to balances the need to provide abstract, safe tasks that are conducive to driving academic research, with operational relevance to ensure that implementations that do well in the competition also represent capabilities that solve real-world challenges. It distills the real world, operational requirements of first responders into elemental tasks. These tasks are a common language, which make it possible to create a benchmark for innovation [6]. Through this language, the challenges of the field are communicated to researchers, in a manner that is clear, easy to reproduce and where all robots can exhibit some level of performance and yet few, if any robots, can saturate. Similarly, the space of capabilities that exist in the research community can be communicated, via their

\footnotetext{
${ }_{1}^{1}$ http://wiki.robocup.org/wiki/Robot_League.

2 US Department of Homeland Security.

${ }^{3}$ US National Institute of Standards and Technology.

${ }^{4}$ Formerly the American Society for Testing and Materials.

5 ASTM International Committee on Homel and Security Applications; Operational Equipment; Robots (E54.08.01).
} 
performance in known tests, to first responders, robot manufacturers and government agencies.

\subsection{The League and Community}

The League extends its efforts to advance the state of response robotics beyond the aforementioned competition. Rescue camps and summer schools [7, 8] disseminate the Best-in-Class capabilities and implementations both within and beyond the League. Participation as organizers and as competitors in other competitions ensure that the experience inside the League spreads more widely.

Besides the world-championship (see Fig. 1 for a group photo) regional competitions are held in several countries, using the same scenarios and rules. Typically only the best teams from the regional opens qualify for the main competitions. Thus the community spans over many more teams then the 20 to 30 teams in the world-championship. Big regional competitions that are open to teams from all regions are regularly held in Germany, Iran, Japan, Thailand and China.

Week-long teaching camps and summer schools, focused on research level undergraduate students, $\mathrm{PhD}$ students and early career researchers, have been hosted by the League community several times since 2004. The first Rescue Robotics Camp was held Italy [9] and was instrumental in not only bringing together and disseminating the Best-in-Class capabilities from the previous year but also to connect the League community more closely with the first responder community.

This theme continued with subsequent events in Thailand, Austria, Turkey and Australia. The 2012 Safety, Security and Rescue Robotics Summer School, held in Alanya, Turkey [10], was unique in that selected senior and retired responders from police bomb squads and fire and rescue services were embedded directly into the different groups for the entire week. This allowed the students to gain a deeper appreciation for the challenges faced by responders in the field. In addition it also allowed the first responders, typically in management and advisory panels of their services, to better understand the current and future state of the art.

\subsection{Lowing the Barrier to Enter the League}

A major challenge is that robots for use in this field must have a combination of mobility, sensing, communications, intelligence, user interface design and software engineering. From its earliest years, the League has been seeking a standard robot platform or kit to lower the barrier for entry to research in this field, especially for computer science based teams who may lack the requisite mechanical engineering expertise to integrate a reliable, high mobility platform. Closing the loop for the first time, on a working robot, is often the greatest challenge. In the past, such teams have resorted to inflexible and proprietary kit robots or toys that lack durability and performance.

With the advent of 3D printing and low-cost smart servos, highly capable embedded computers and other such resources, starting in 2014 the League has started the Open Academic Robot $\mathrm{Kit}^{6}$ [11]. This is an initiative to develop a family of robot designs where all mechanical parts are 3D printable, All other parts are readily available off the shelf and all designs, instructions and source code are available in easily editable form, online, under an open source license. Furthermore, the parts are, where possible, drawn from a common set of parts to maximize potential re-use. The aim is to generate a set of online resources that anyone can follow to create a working robot that they can then improve within their area of expertise. To complement the smaller robots that tend to be constructed using 3D printing, the League is also launching the Rapidly Manufactured Robot League, a competition designed for robots in confined spaces, as described in Sect. 2.4.3. The first two robots from this initiative are shown in Fig. 2. These initial designs have also focused on being low in cost. At approximately $\$ 500$ USD, they are comparable to many moderately advanced robotics construction kits and yet they are already complete with onboard cameras, computation via a Raspberry Pi embedded computer, and a user interface that can be controlled from an Android device.

Teams around the world, all working on similar open source robots, can contribute improvements to a common pool and thus form ad-hoc collaborations regardless of their location or their stage of education. For example, high school students in Thailand might generate new wheel designs while graduate students in Germany could design vision algorithms for recognizing impassable terrain. A team from a makerspace in Australia might then design a new gripper while an undergraduate team from the United States could build a new user interface. All of these improvements can be shared and these groups connected via the kit, long before they may meet at a competition or teaching event.

\subsection{Additional Test Elements}

\subsubsection{Aerial Robots}

Aerial vehicles are of tremendous use in response robot scenarios and are widely used already today. But their application is mostly on wide-area surveying, mapping and search. But Unmanned Aerial Vehicles (UAV) also have a great potential for search and inspection close to or inside

\footnotetext{
${ }^{6}$ http://www.oarkit.org.
} 


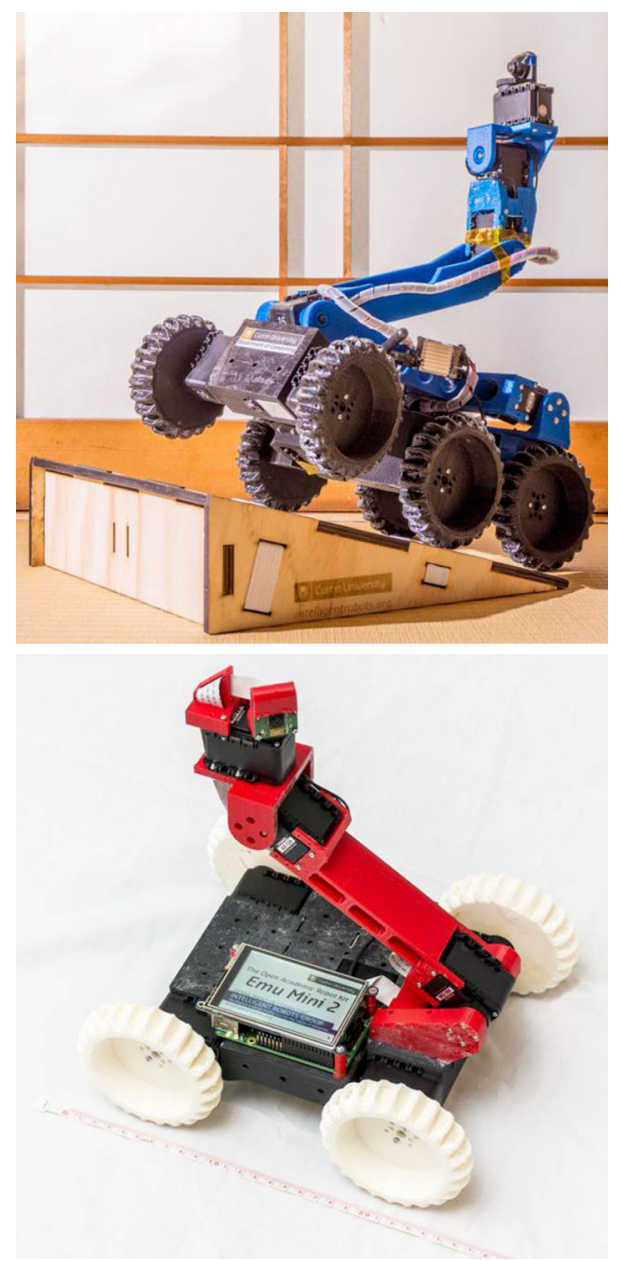

Fig. 2 The "excessively complex six-wheeled robot" (top) and the "Emu Mini 2" (bottom), the first two robots from the Open Academic Robot Kit

of buildings. The RRL recognizes the opportunities and challenges of this use of UAVs. A number of standard test methods for aerial vehicles are installed in the aerial arena which is part of the overall arena. Safety features (e.g. low battery and communication loss behaviors) as well as specialized capabilities (e.g. building access through windows, station keeping) are tested for the Best in Class MicroAerial Robot.

\subsubsection{Outdoor Robots}

In 2016 for the first time an outdoor competition is organized which is affiliated to the Robot Rescue League. In this CarryBot league cheap and simple, but yet capable, autonomous robots for basic logistic purposes are tested. The goal is to support the response personnel by transporting material or even victims over moderately difficult terrain along a path predefined with GPS coordinates.

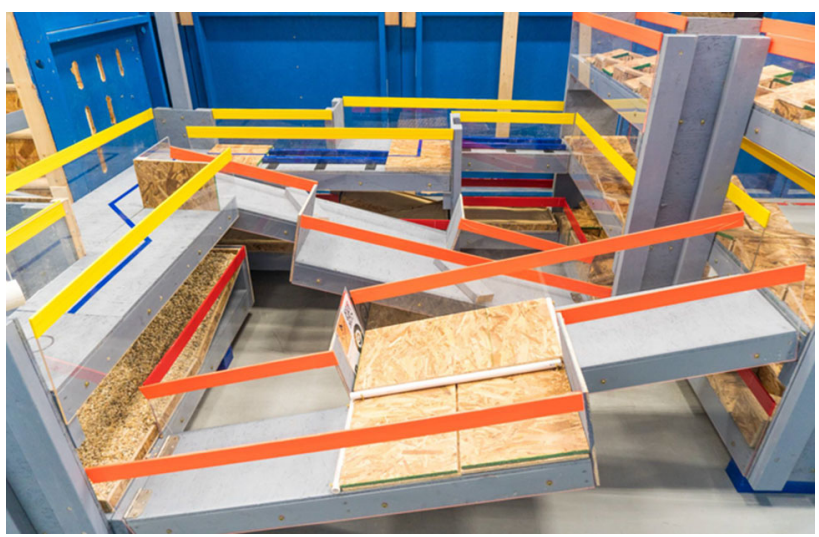

Fig. 3 The smaller scale rapidly manufactured Robot League arena, based on a $30 \mathrm{~cm}(1 \mathrm{ft})$ nominal clearance

\subsubsection{Confined Space Robots}

The scale of robots that compete in the RoboCup Rescue Robot League are designed to enter spaces with a nominal

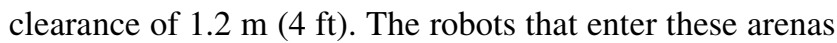
are very capable; however they also tend to be very expensive and complex. Furthermore, there is a demand for robots that can operate in significantly smaller spaces, as are found in collapsed buildings and other industrial, civil and domestic environments. To encourage the development of smaller robots, and to allow cheaper robots such as those of the Open Academic Robot Kit to compete on their own terms, since 2014 the League has developed a bracket of the competition for smaller robots. Named the Rapidly Manufactured Robot League (also referred to as the Mini Arena and formerly the Confined Space Challenge), this arena is based on a $30 \mathrm{~cm}(1 \mathrm{ft})$ nominal clearance. This arena is shown in Fig. 3.

Reducing the size of the arena and thus also reducing the cost of the robots required also allows the League to reach across to the RoboCup Junior Rescue community. The existing RoboCup Junior Rescue arenas are already based on a maze at a scale of $30 \mathrm{~cm}(1 \mathrm{ft})$. The Rapidly Manufactured Robot League provides a bridge competition that allows high school students to tackle research level problems in mechanics, electronics, computer science, and user interfaces, at a cost and level of required infrastructure that is similar to their existing competitions.

\subsection{Technological Developments and Lessons Learned}

Recent years have seen several improvements in the technology employed by robotic rescue systems. Those improvements are then often met with more challenging tests in the RoboCup Rescue competition. 
A development that can be easily overlooked is the gradually increasing difficulty in the terrain that the autonomous robots have to face. Over the years we went from mostly flat terrain to crossing ramps. We also introduced shortcuts into the more difficult orange arena that require robots with simple locomotion to detect that the terrain is impassible for them. In 2015 obstacles that require $3 \mathrm{D}$ terrain classification were introduced as well as curtains made of light fabric that require advanced sensing and reasoning skills from the autonomous robot.

Also the manipulation capabilities of the robots have improved. As a consequence we now have multiple doors in the arena that can be opened in push as well as pull direction. Further improvements are that two-way audio communication is now required. On the operation side we are now including the setup time of the operator station in the run time and also restrict the size of the operator station. Thus the teams are pushed to more ergonomic and easy to use human-robot interfaces.

Another aspect is the adjudication of the league in itself poses interesting scientific questions. The development of the standard test methods is one such area. Mapping and the evaluation of the generated maps is another research area that is important for the league. The Fiducial method for 2D grid map evaluation [12] has recently been extended to 3D maps, using data from the RoboCup Rescue competition [13].

\subsection{Influence Outside the League}

Members from the League community were extensively involved in the DARPA Robotics Challenge (DRC) Trials and Finals [14]. The challenges seen in the DRC Trials were developed by members of the League organizing committee while one of the teams that qualified for the finals, Team ViGIR [15], consisted of many members of Team Hector, one of the most successful teams in the League. Similar principles were used to develop the challenges in the DRC Trials as the test methods and the RoboCupRescue Robot League. These focus on test apparatuses that are easy to build, yield statistically significant results, exercise operationally relevant capabilities and that are easy for suitable robots to attempt and yet can challenge even the most capable robots. Early concepts for apparatuses such as valves and terrains were evaluated in the RoboCupRescue Robot League prior to their final appearance at the DARPA Robotics Challenge.

In 2016, RoboCupRescue will welcome humanoid robots with challenges that are an evolution of those that appeared in the DRC Finals. This crossover aims to showcase advances in human technologies in disaster scenarios, provide an evolving benchmark for disaster relief that requires more dexterity than standard wheeled robots, recruit new teams and leverage the investment that the research community has made in the DRC efforts. The initial set of tasks for this demonstration would focus on using human tools in human environments-analogous to the @Home league. The task environment, however, would replicate aspects of the Rescue league.

\section{Rescue Simulation}

The RoboCup Rescue Simulation League (RSL) aims to develop simulators that form the infrastructure of the simulation system and emulate realistic phenomena predominant in disasters and it aims to develop intelligent agents and robots that are given the capabilities of the main actors in a disaster response scenario.

The RoboCup Simulation League has two major competitions which will be described in the subsequent sections. The two competitions share the Infrastructure competition, which is intended to stimulate the further development of the league with new challenges. Champions of the league are recognized at the League's wiki ${ }^{7}$ and get the chance to publish their contribution $[16,17]$ in the Springer Lecture Notes series.

A prequel of the DARPA Robotics Challenge Field Trials was the Virtual Robotics Challenge [18], with nearly 100 teams participating. This humanoid challenge was based on a dedicated version of the Gazebo simulator, which in 2016 also has become the basis of the RoboCup Rescue Virtual Robot competition [19].

\subsection{Virtual Robot Competition}

RoboCup Virtual Robot competitions are being held since 2006 [20]. The intention of the competition was to create a bridge between the RRL and RSL [21]. The competition attracts mainly academic teams from universities, some even with teams competing in both the RoboCup Rescue Robot and Simulation League. In 2016 the competition reached across and attracted high school teams with prior experience in the RoboCup Junior Rescue community; performing precisely the bridging function intended for the Rapidly Manufactured Robot League.

The main challenge for the teams is the control of a large team of robots (typically eight) by a single operator. This is still state-of-the art; the only real comparison is the champion of the Magic competition [22], where 14 robots were controlled by two operators. In simulation it was demonstrated that a single operator is able to control a maximum of 24 robots [23].

\footnotetext{
${ }^{7}$ http://wiki.robocup.org/wiki/Rescue_Simulation_League.
} 
Fig. 4 Impressions of the Virtual Robot Competition in 2006 and 2008
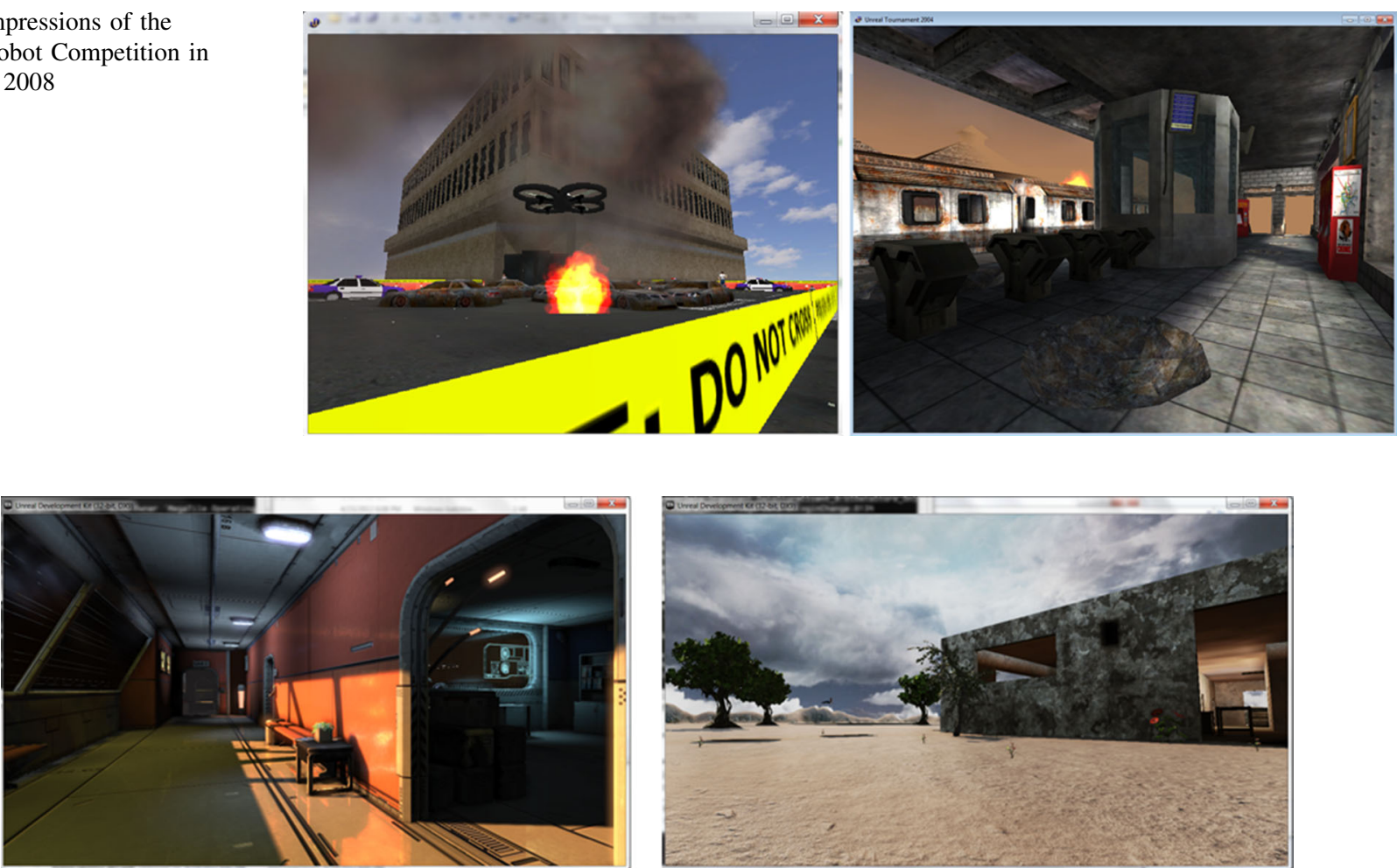

Fig. 5 Impressions of the Virtual Robot Competition in 2012 \& 2014

The single operator has to use high-level commands (such as the areas to be searched, routes to be followed, etc.) to be able to control such large teams [24]. The operator's attention is mostly needed to verify observations whether or not one of the robots has detected a victim (based on color and/or shape). Due to poor lighting and the number of occlusions, the conditions are generally not favorable for automatic victim detection, and manual conformation is always needed. The approach to a victim is quite critical [(the robot should come within the communication range $(<1 \mathrm{~m})$ ], but is not allowed to touch the body or any of the limbs). This means that the workload for the operator is quite high, providing an advantage for the teams which are able to automate the decision making within the robot team as far as possible, and only involve the operator when needed.

The shared map generated by the robots during the competition has a central role in the coordination of such large robot teams. The shared map is where the distributed sensor information is collected and registered, by each robot independently. The information has to be sent via often unreliable communication links [25], so the robot has selected which information is to be broadcasted (the robots have a need to know what could be of interest for its teammates and the operator). The registration process is asynchronous; some information may arrive at the basestation even minutes after the actual observation [26].
There is no guarantee that the operator has time to look at this information directly, which implies that the map within the user interface has to be interactive and should allow the operator to call back observations that were made at any point of interest (independent of when the observation was made and by which robot). At the same time the registration process should keep the map clean (no false positives or wrong associations), because it is the area where the coordination of the team behaviors is done.

Since the beginning of the competition [20], a number of challenging disaster environments have been created. Already at the RoboCup 2006 a quite large world was used, which had a street scenario, an office scenario and a hedge maze in the garden, as illustrated in Fig. 4. In later competitions a large disaster area with a railway station at a waterfront was used. These environments were based on the Unreal Engine 2 (UT2004).

With the introduction of the Unreal Engine 3 (UDK) even larger and more detailed environments could be created. For instance, in the 2012 competition a world with very dynamic lighting with moving shadows was introduced, as illustrated in Fig. 5). In 2014 the outdoor worlds were already so large that only teams of combined air- and ground-robots could explore the disaster site.

To be able to control explore these large environments not only improvements of the user interface for the operator were needed, but the teams also increased the 
autonomy of the robots. For instance, several well-known methods [27, 28] were applied to be able to automatically recognize victims [29, 30].

To make pure teleoperation of robots based on visual feedback more difficult, indoor environments were often filled with smoke (which is realistic in disaster scenarios [31]). To counter this situation, the teams used methods [32] to increase the contrast in smoky and dark circumstances, which is also valuable for first responders.

Many publications related to this competition were published, some with quite high impact [21, 33, 34]. The subjects were as diverse as walking robots, design of test arenas and mapping algorithms.

\subsection{Agent Competition}

The goal of the RoboCup Rescue Agent competition is to compare the performance of different algorithms for coordinating and controlling a team of physical agents performing disaster mitigation in a simulated city [35]. The goal of teams participating in the competition is to provide a software system that reacts to a simulated disaster situation by coordinating a group of agents. This goal leads to challenges such as the exploration of large-scale environments in order to localize fire-fronts and victims, as well as the scheduling of time-critical rescue missions. Agents have only a limited amount of communication bandwidth they can use to coordinate with each other [36]. The problem cannot be addressed by a single entity, but has to be solved by a multi-agent system. Moreover, the simulated environment is highly dynamic and only partially observable by a single agent. Agents have to plan and decide their actions asynchronously in real-time.

The agent competition consists of a simulation platform which resembles a city after an earthquake. Such a simulation of the city of Kobe is depicted by Fig. 6. Into this

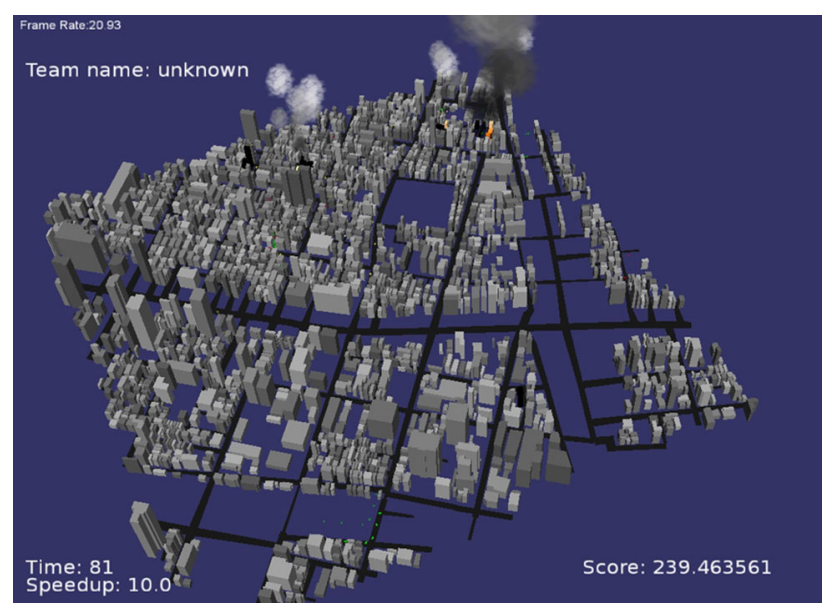

Fig. 6 A simulation of the city of Kobe burning environment, intelligent agents can be spawned for mitigating the effects of simulated disaster events, such as flood and fire. For this purpose, agents may take on heterogeneous roles such as police force, fire brigade, and ambulance team, that all have different capabilities.

Several overview articles are written on the coordination and task allocation research performed with the RoboCup Rescue Agent simulator [37, 38]. As indicated by Ferreira et al. [39], generic algorithms tend to be outperformed the methods applied by the winners of the RoboCup Rescue Agent competition [40], which use various heuristics based on a-priori knowledge on the domain.

Inspired by the influential paper by Murphy et al. [41] on physical rescue agents, several researchers have applied their knowledge in real disaster situations [42-44]. Most important, as implemented as task for the ambulance agents in the Rescue Simulation Agent competition, is to reduce the amount of time a victim is entrapped.

Within the last years, there were several techniques for multi-agent strategy planning and team coordination introduced, such as decentralized communicating POMDPs [45], distributed constraint optimization [46], auction based methods [47] and evolutionary learning [48, 49]. Recently, this was extended with work on weighted synergy graphs [50], Tractable Higher Order Potentials constraints [51] and fluid team allocations [52]. Furthermore, there has been substantial work on building information infrastructure and decision support systems for enabling incident commanders to efficiently coordinate rescue teams in the field [53].

\section{New Challenges}

\subsection{Rescue Simulation League}

In 2013 the simulation league has initiated RMasBench, a new type of challenge having the goal to focus on the strategic decisions instead of the tactical decisions [54]. The idea is to extract from the entire problem addressed by the agents certain aspects such as task allocation, team formation, and route planning, and to present these sub problems in an isolated manner as stand-alone problem scenarios with an abstract interface. As a consequence, participating teams are more free to focus on their research without having to deal with low-level issues. RMasBench introduced a generic API for distributed constraint optimization problem (DCOP) algorithms, including a library implementing state-of-the-art DCOP solvers, such as DSA and MaxSum as reference solutions. In 2016 the challenge is rephrased as Technical Challenge, which the same intention to abstract away from the low-level tactical decisions, but this time facilitated by an Agent Develop Framework [55]. 


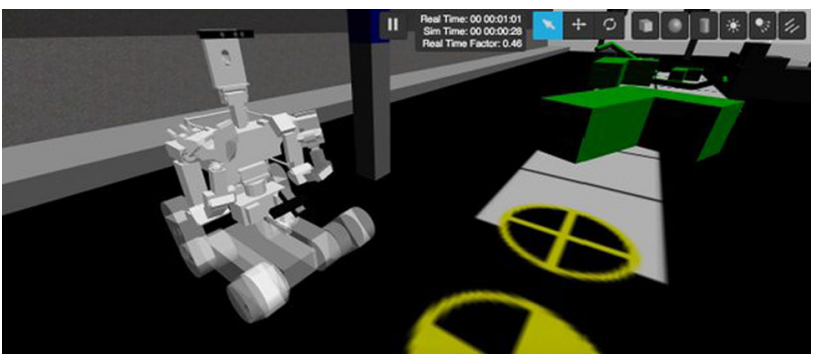

Fig. 7 Team Hector's Centaur at the JVRC's 'clear the road' task

The future of the Virtual Robot competition was redefined at the Future of Rescue Simulation workshop. One of the goals of the workshop was to define a roadmap for the development of the league for the coming years. In addition a connection to the DARPA Virtual Robot Challenge and the Japanese Virtual Robot Challenge (JVRC) [56] was made. At the JVRC a centaur design was very successful; a tracked robot with a humanoid torso called MIDJAXON. The centaur design could also be a good combination of mobility and manipulation capabilities in the RoboCup Virtual Robot competition, as demonstrated at the workshop ${ }^{8}$ (See Fig. 7).

As a result of the workshop the challenge of the Virtual Robot Competition is refined, which is reflected in a new rules document [57]. In this new rules document a clear transition is made from the current Unreal/ROS based environment [58] towards a ROS/Gazebo based environment [59].

\subsection{Robot Rescue League}

Starting in 2016 the RRL is adopting a new scheme for the competition. In the preliminary rounds in the first three days basic and specific capabilities of the robots are measured in DHS-NIST-ASTM International Standard response robot test apparatuses, for which the testing procedures have been customized to the specific needs of the RoboCup Real Rescue League. The test method apparatuses will be arranged into lanes and teams will be invited to run their robots multiple times across the lanes. By running these tests in parallel, rigorous measurements of capabilities can be obtained in isolation. This results in statistically significant testing in four areas: (1) basic sensing and MANeuvering capabilities, (2) advanced MOBility, (3) manipulation and inspection DEXterity and (4) EXPloration, mapping and autonomy. Each of those four areas consists of five tests, which often correspond to one of the standard ASTM test methods. Figure 8 shows an overview of how the tests are laid out in the arena.

\footnotetext{
${ }^{8}$ https://github.com/tu-darmstadt-ros-pkg/centaur_robot_tutorial/wiki.
}

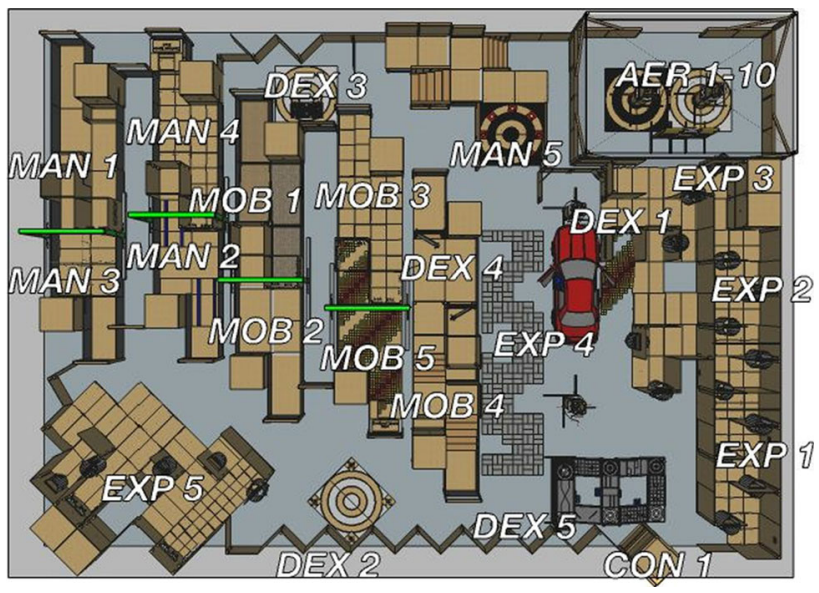

Fig. 8 Real Rescue arena plan for 2016

In the preliminaries the Best-in-Class winners in the areas of mobility, dexterity and exploration will be determined as well as the overall best teams that will progress to the finals. For each area three Best-in-Class certificates are awarded: Best-in-Class Small Robot (for robots entering the tests through a $60 \mathrm{~cm}$ square), Best-in-Class Autonomous Robots (for robots performing without operator intervention) and the general Best-in-Class certificates open to all teams.

In the finals the test elements will be combined such that two big arenas are formed. The finalists will then search in there for simulated victims by traversing the various test elements within a single run.

Running the competition with this new scheme enables us to conduct challenging and fair competitions that emphasize tasks that are of actual value for USAR applications. The RRL is now more closely resembling Response Robot Exercises [60], which have been effective in communicating and demonstrating functionality, reliability, operator proficiency, and autonomous/assistive capabilities of the systems between robot manufacturers and responders.

As part of the emphasis on the dissemination and collaborative development of technologies for response robotics, from 2016 on the Team Description Papers (TDP) have an updated template covering more technical aspects of the robotic solutions. The goal of this update is to better allow teams to express and share the novel aspects of their entries. The TDPs of all participating teams will be published online ${ }^{9}$ and thus be accessible to the general public.

The new rules have been tested and implemented at the RRL meeting in March 2016 in Koblenz, Germany and at the Iran Open in April 2016. More details about the new way the league is run can be found in the rules document [61].

\footnotetext{
${ }^{9}$ http://www.robocuprescue.org/.
} 


\section{Conclusions}

In the last 16 years the RoboCup Rescue community has proven that work on this grand challenge [2] is fruitful. Teams from all over the world are now working on this socially relevant application, evolving their initial hardware designs to very versatile robots. Also the perception, planning and control of the robots have been substantial improved, which makes it possible to autonomously navigate through the disaster area and find victims in difficult circumstances. The rescue robots are no longer alone, but operate in heterogeneous teams combining robots with different capabilities. Coordination inside the team of robots, so that they efficiently work on a joint goal, is extensively studied in simulation and demonstrated with real robots.

One of the remaining challenges for the coming years is the manipulation capabilities. The DARPA Robotics Challenge has proven that humanoid robots could make use of available tools (cars, drills, valves) in their rescue missions, but it is also clear that there could be a lot improved in manipulation capabilities. Yet, in the future the rescue robots should not only be able to find the victims but also capable to free them carefully from their perilous situation.

Open Access This article is distributed under the terms of the Creative Commons Attribution 4.0 International License (http://crea tivecommons.org/licenses/by/4.0/), which permits unrestricted use, distribution, and reproduction in any medium, provided you give appropriate credit to the original author(s) and the source, provide a link to the Creative Commons license, and indicate if changes were made.

\section{References}

1. Tadokoro S, Kitano H, Takahashi T, Noda I, Matsubara H, Shinjou A, Koto T, Takeuchi I, Takahashi H, Matsuno F, Hatayama M, Nobe J, Shimada S (2000) The RoboCup-Rescue project: a robotic approach to the disaster mitigation problem. In: Proceedings of the IEEE Int. Conf. on robotics and automation (ICRA)

2. Kitano H, Tadokoro S (2001) A grand challenge for multiagent and intelligent systems. AI Mag 22:39-52

3. Murphy RR, Tadokoro S, Nardi D, Jacoff A, Fiorini P, Choset H, Erkmen AM (2008) Search and rescue robotics. In: Springer handbook of robotics. Springer, Berlin, pp 1151-1173 (ISBN 978-3-540-30301-5)

4. Kohlbrecher S, Meyer J, Graber T, Petersen K, Klingauf U, Stryk O (2014) Hector open source modules for autonomous mapping and navigation with rescue robots. In: RoboCup 2013: Robot World Cup XVII. Springer, Berlin, pp 624-631 (ISBN 978-3662-44468-9)

5. Jacoff A, Sheh R, Virts AM, Kimura T, Pellenz J, Schwertfeger S, Suthakorn J (2012) Using competitions to advance the development of standard test methods for response robots. In: Proceedings of the workshop on performance metrics for intelligent systems, ACM, pp 182-189
6. Amigoni F, Bastianelli E, Berghofer J, Bonarini A, Fontana G, Hochgeschwender N, Iocchi L, Kraetzschmar G, Lima P, Matteucci M, Miraldo P, Nardi D, Schiaffonati V (2015) Competitions for benchmarking: task and functionality scoring complete performance assessment. IEEE Robot Autom Mag 22(3):53-61 (ISSN 1070-9932)

7. Sheh R, Komsuoglu H (2012) The 2012 ieee robotics and automation society safety, security, and rescue robotics summer school: an event for the dissemination of the challenges and bestin-class capabilities in the ssrr community (society news). IEEE Robot Autom Mag 19(4):92-95 (ISSN 1070-9932)

8. Sheh R, Collidge B, Lazarescu M, Komsuoglu H, Jacoff A (2014) The response robotics summer school 2013: bringing responders and researchers together to advance response robotics. In: Intelligent robots and systems (IROS 2014), 2014 IEEE/RSJ international conference on, IEEE, pp 1862-1867

9. Scholtz Jean (2005) Robot rescue camp. Interactions 12(2):79-80

10. Sheh R, Komsuoğlu H, Jacoff A, Kimura T, Nardi D, Pellenz J, Steinbauer G (2012) The 2012 safety, security, and rescue robotics summer school. In: IEEE international symposium on safety, security, and rescue robotics (SSRR), 2012, pp 1-2

11. Sheh R, Komsuoglu H, Jacoff A (2014) The open academic robot kit: lowering the barrier of entry for research into response robotics. In: Proceedings of the 2014 IEEE international symposium on safety, security, and rescue robotics (SSSR), pp 1-6

12. Schwertfeger S, Jacoff A, Pellenz J, Birk A (2011) Using a fiducial map metric for assessing map quality in the context of robocup rescue. In: International workshop on safety, security, and rescue robotics (SSRR), pp 208-214

13. Schwertfeger S, Birk A (2015) Using fiducials in 3d map evaluation. In: IEEE international symposium on safety, security, rescue robotics (SSRR)

14. Guizzo E, Ackerman E (2015) The hard lessons of darpa's robotics challenge (news). IEEE Spect 52(8):11-13, (ISSN 0018-9235)

15. Kohlbrecher S, Romay A, Stumpf A, Gupta A, von Stryk O, Bacim F, Bowman DA, Goins A, Balasubramanian R, Conner DC (2015) Human-robot teaming for rescue missions: Team ViGIR's approach to the 2013 DARPA robotics challenge trials. J Field Robot 32(3):352-377. doi:10.1002/rob.21558

16. Amigoni F, Visser A, Tsushima M (2013) RoboCup 2012 rescue simulation league winners. In: RoboCup 2012: Robot Soccer World Cup XVI. Springer, Berlin, pp 20-35 (ISBN 978-3-64239250-4)

17. Spirin V, de Hoog J, Visser A, Cameron S (2015) MRESim, a multi-robot exploration simulator for the rescue simulation league. In: Bianchi C, Reinaldo A, Akin LH, Ramamoorthy S, Sugiura K (eds) RoboCup 2014: Robot World Cup XVIII. Springer, Cham, pp 106-117. doi: 10.1007/978-3-319-18615-3_ 9. (ISBN 978-3-319-18615-3)

18. Aguero Carlos E, Koenig Nate, Chen Ian, Boyer Hugo, Peters Steven, Hsu John, Gerkey Brian, Paepcke Steffi, Rivero Jose L, Manzo Justin et al (2015) Inside the virtual robotics challenge: simulating real-time robotic disaster response. IEEE Trans Autom Sci Eng 12(2):494-506

19. Visser A, Shimizu M, Koenig N, Takashi T (2016) A realistic RoboCup rescue simulation based on Gazebo. In: RoboCup 2015: Robot World Cup XIX, pp 331-338 (ISBN 978-3-319-29338-7)

20. Balakirsky S, Scrapper C, Carpin S, Lewis M (2006) Usarsim: providing a framework for multi-robot performance evaluation. In: Proceedings of PerMIS

21. Carpin S, Wang J, Lewis M, Birk A, Jacoff A (2006) High fidelity tools for rescue robotics: results and perspectives. In: RoboCup 2005: Robot Soccer World Cup IX. Springer, Berlin, pp 301-311

22. Olson Edwin, Strom Johannes, Morton Ryan, Richardson Andrew, Ranganathan Pradeep, Goeddel Robert, Bulic Mihai, Crossman Jacob, Marinier Bob (2012) Progress toward multi- 
robot reconnaissance and the magic 2010 competition. J Field Robot 29(5):762-792

23. Wang H, Chien SY, Lewis M, Velagapudi P, Scerri P, Sycara K (2009) Human teams for large scale multirobot control. In: IEEE international conference on systems, man and cybernetic (SMC), pp 1269-1274

24. Caltieri A, Amigoni F (2013) High-level commands in humanrobot interaction for search and rescue. In: RoboCup 2013: Robot World Cup XVII. Springer, Berlin, pp 480-491

25. Banfi J, Li AQ, Basilico N, Amigoni F (2015) Communicationconstrained multirobot exploration: short taxonomy and comparative results. In: Proceedings of the IROS workshop on on-line decision-making in multi-robot coordination (DEMUR2015), pp 1-8

26. Spirin V, Cameron S, de Hoog J (2013) Time preference for information in multi-agent exploration with limited communication. In: Towards autonomous robotic systems. Springer, Berlin, pp 34-45

27. Viola P, Jones M (2001) Rapid object detection using a boosted cascade of simple features. In: Computer vision and pattern recognition, 2001. CVPR 2001. Proceedings of the 2001 IEEE Computer Society Conference on, vol 1, pp I-511, IEEE

28. Dalal N, Triggs B (2005) Histograms of oriented gradients for human detection. In: Computer vision and pattern recognition, CVPR 2005. IEEE computer society conference on, vol 1, pp 886-893. IEEE

29. Flynn H, De Hoog J, Cameron S (2009) Integrating automated object detection into mapping in usarsim. In: Proceedings of the international conference on intelligent robots and systems (IROS 2009), Workshop on robots, games, and research: success stories in USARSim, pp 29-34. Citeseer

30. Uzun Y, Balcilar M, Mahmoodi K, Davletov F, Amasyali MF, Yavuz S (2013) Usage of hog (histograms of oriented gradients) features for victim detection at disaster areas. In: Electrical and Electronics Engineering (ELECO), 2013 8th International Conference on pp 535-538

31. Formsma O, Dijkshoorn N, van Noort S, Visser A (Springer) Realistic simulation of laser range finder behavior in a smoky environment. In: RoboCup 2010: Robot Soccer World Cup XIV. Springer, Berlin, pp 336-349

32. Bogdanova V (2010) Image enhancement using retinex algorithms and epitomic representation. Bulg Acad Sci Cybern Inform Technol 10(3):10-19

33. Zaratti M, Fratarcangeli M, Iocchi L (2006) A 3d simulator of multiple legged robots based on usarsim. In: Robocup 2006: Robot Soccer World Cup X. Springer, Berlin, pp 13-24

34. Pfingsthorn M, Slamet B, Visser A (2007) A scalable hybrid multi-robot slam method for highly detailed maps. In: RoboCup 2007: Robot Soccer World Cup XI. Springer, Berlin, pp 457-464

35. Kitano H, Tadokoro S, Noda I, Matsubara H, Takahashi T, Shinjou A, Shimada S (1999) RoboCup Rescue: search and rescue in large-scale disasters as a domain for autonomous agents research. In: IEEE Conf. on Man, Systems, and Cybernetics (SMC-99)

36. Rooker MN, Birk A (2004) Combining exploration and ad-hoc networking in robocup rescue. In: RoboCup 2004: Robot Soccer World Cup VIII. Springer, Berlin, pp 236-246

37. Ramchurn Sarvapali D, Farinelli Alessandro, Macarthur Kathryn S, Jennings Nicholas R (2010) Decentralized coordination in robocup rescue. Comput J 53(9):1447-1461

38. Jia X, Meng MQ-H (2013) A survey and analysis of task allocation algorithms in multi-robot systems. In: Robotics and biomimetics (ROBIO), 2013 IEEE international conference on, pp 2280-2285. IEEE

39. Jr Paulo Roberto Ferreira, Dos Santos Fernando, Bazzan ALC, Epstein D, Waskow SJ (2010) Robocup rescue as multiagent task allocation among teams: experiments with task interdependencies. Auton Agents MultiAgent Syst 20(3):421-443

40. Morimoto T, Kono K, Takeuchi I (2001) Yabai the first rescue simulation league champion. In: RoboCup 2001: Robot Soccer World Cup V. Springer, Berlin, pp 49-59

41. Murphy RR, Casper J, Micire M (2000) Potential tasks and research issues for mobile robots in robocup rescue. In: RoboCup 2000: Robot Soccer World Cup IV. Springer, Berlin, pp 339-344

42. Linder T, Tretyakov V, Blumenthal S, Molitor P, Holz D, Murphy R, Tadokoro S, Surmann H (2010) Rescue robots at the collapse of the municipal archive of cologne city: a field report. In: IEEE international workshop on safety security and rescue robotics (SSRR), pp 1-6

43. Kruijff GJM, Tretyakov V, Linder T, Pirri F, Gianni M, Papadakis P, Pizzoli M, Sinha A, Pianese E, Salvatore Corrao, et al. Rescue robots at earthquake-hit mirandola, italy: a field report. In: Safety, security, and rescue robotics (SSRR), 2012 IEEE international symposium on, pp 1-8. IEEE

44. Matsuno F, Sato N, Kon K, Igarashi H, Kimura T, Murphy R (2014) Utilization of robot systems in disaster sites of the great eastern japan earthquake. In: Field and service robotics. Springer, Berlin, pp 1-17

45. Nair R, Tambe M, Marsella S (2002) Team formation for reformation in multiagent domains like robocuprescue. In: RoboCup 2002: Robot Soccer World Cup VI. Springer, Berlin, pp 150-161

46. Scerri P, Farinelli A, Okamoto S, Tambe M (2005) Allocating tasks in extreme teams. In: Proceedings of the fourth international joint conference on Autonomous agents and multiagent systems, pp 727-734. ACM

47. Sedaghat MN, Nejad LP, Iravanian S, Rafiee E (2005) Task allocation for the police force agents in robocuprescue simulation. In: RoboCup 2005: Robot Soccer World Cup IX. Springer, Berlin, pp 656-664

48. Martínez I, Ojeda D, Zamora E (2007) Ambulance decision support using evolutionary reinforcement learning in robocup rescue simulation league. In: RoboCup 2006: Robot Soccer World Cup X, pp 556-563

49. Dos Santos Fernando, Bazzan Ana LC (2011) Towards efficient multiagent task allocation in the robocup rescue: a biologicallyinspired approach. Auton Agents MultiAgent Syst 22(3):465-486

50. Liemhetcharat Somchaya, Veloso Manuela (2014) Weighted synergy graphs for effective team formation with heterogeneous ad hoc agents. Artif Intell 208:41-65

51. Pujol-Gonzalez M, Cerquides J, Farinelli A, Meseguer P, Rodriguez-Aguilar JA (2015) Efficient inter-team task allocation in robocup rescue. In: Proceedings of the international conference on autonomous agents and multiagent systems, pp 413-421,

52. Parker J, Nunes E, Godoy J, Gini M (2015) Exploiting spatial locality and heterogeneity of agents for searchand rescue teamwork. J Field Robot. doi:10.1002/rob.21601

53. Schurr N, Marecki J, Lewis, Tambe M, Scerri P (2005) The defacto system: coordinating human-agent teams for the future of disaster response. In: Multi-agent programming: languages, platforms and applications. Springer, Boston, pp 197-215 (ISBN 978-0-387-26350-2)

54. Kleiner A, Farinelli A, Ramchurn S, Shi B, Maffioletti F, Reffato R (2013) Rmasbench: benchmarking dynamic multi-agent coordination in urban search and rescue. In: Proceedings of the 2013 international conference on Autonomous agents and multi-agent systems. International Foundation for Autonomous Agents and Multiagent Systems, pp 1195-1196

55. Takayanag K, Takami S, Kozuka Y, Ito N (2015) Design and implementation of the agent framework for the robocup rescue simulation new entrants. In: Proceedings of the he 29th annual conference of the Japanese Society for Artificial Intelligence. 2B5-NFC-02c-2 
56. Okugawa M, Oogane K, Shimizu M, Ohtsubo Y, Kimura T, Takahashi T, Tadokoro S (2015) Proposal of inspection and rescue tasks for tunnel disasters??? task development of japan virtual robotics challenge. In: 2015 IEEE international symposium on safety, security, and rescue robotics (SSRR), pp 1-2

57. Francesco A, Masaru S, Shimizu T, Arnoud V (2016) RoboCup 2016 - RoboCup Rescue Simulation League - Virtual Robot Competition - Rules Document. https://staff.fnwi.uva.nl/a.visser/ activities/robocup/RoboCup2016/RoboCup2016_Rules_v1.5.pdf

58. Kootbally Z, Balakirsky S, Visser A (2014) Enabling codesharing in rescue simulation with usarsim/ros. In: RoboCup 2013: Robot World Cup XVII, vol 8371 of Lecture notes in computer science. Springer, Berlin, pp 592-599
59. Shimizu M, Koenig N, Visser A, Takashi T (2015) A realistic robocup rescue simulation based on gazebo. In: RoboCup Symposium 2015, development track

60. Jacoff A, Messina E, Huang HM, Virts A, Downs A, Norcross R, Sheh R (2014) Guide for evaluating, purchasing, and training with response robots using DHS-NIST-ASTM international standard test methods. National Institute of Standards and Technology report

61. RoboCupRescue Organising Committee (2016) RoboCup Rescue Rulebook Version 1.0 